\title{
0422096 12pgs
}

\section{CRRES OBSERVATIONS OF PARTICLE FLUX DROPOUT EVENTS}

\author{
J. Fennell ${ }^{1}$, J. Roeder ${ }^{1}$, H. Spence ${ }^{2}$, H. Singer ${ }^{3}$, A. Korth ${ }^{4}$, M. Grande $^{5}$, and A. Vampola 6 \\ TECH. RPT. NAGW- 453Y \\ IThe Aerospace Corporation, P.0. Box 92957 Los Angeles, CA, 90009 USA \\ $1 N-46$ \\ 2 Boston University, Boston, MA 02215, USA \\ ${ }^{3}$ NOAA R/E/SE, Boulder, CO 80303, USA \\ ${ }^{4}$ Max Planck Institule for Aeronomy, Lindau, Germany

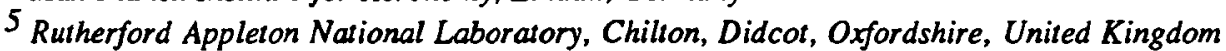 \\ 6P. O. Box 10225, Torrance, CA 90505 USA
}

\begin{abstract}
The complete disappearance of energetic electrons was observed by CRRES in the near geosynchronous region in $7.5 \%$ of the orbits examined. These total flux dropouts were defined by the fluxes rapidly dropping to levels below the sensitivity of the MEA energetic electron spectrometer on the CRRES satellite. They were separated into those that were only energetic electron dropouts and those that were associated with energetic ion and plasma dropouts. Approximately $20 \%$ of the events showed dropouts of all particle fluxes, and these were usually coincident with large increases in the local magnetic intensity and signatures of strong current systems. The energetic particle instruments and magnetometer on CRRES provide a detailed picture of the particle and field responses to these unusual conditions. Both the local moming and dusk events were associated with strong azimuthal (eastward) and radial changes in the magnetic field indicative of a strong current system approaching and sometimes crossing the CRRES position at the time of the flux dropouts. The direction of the field changes and the details of particle observations are consistent with CRRES passing through the plasma sheet boundary layer and entering the tail lobe for a significant number of the events.
\end{abstract}

\section{INTRODUCTION}

Changes in the energetic particle distributions at near geosynchronous altitudes are often observed in association with substorm processes and the large scale boundary motions that often accompany them /1/. For example, on the dayside, the compression of the magnetosphere by solar wind pressure pulses can move the magnetopause inside the geostationary orbit $/ 2,3 /$. This is evidenced by the "dropout" of energetic particle fluxes observed by spacecraft in the dayside magnetosphere $/ 2,3,4 /$. This occurs because the interplanetary energetic particle fluxes are orders of magnitude lower than those found inside the magnetosphere. Thus, when the boundary is "pushed" earthwards of a magnetospheric satellite's orbital position, it changes from observing high magnetospheric fluxes to observing the low interplanetary flux in a very short time (i.e., the flux drops out). It is relatively uncommon to observe such magnetopause crossing events near geosynchronous orbit $/ 2 /$. In the nightside magnetosphere, the distortion of the geomagnetic field caused by the intensified cross-tail currents which arise during the substorm growth phase can cause significant modification in the energetic particle angular distributions and intensities. These can become so extreme as to cause complete flux dropouts of the energetic particles (see for example refs. $/ 5 /$ and $/ 6 /$ ). Normally, such signatures are observed in the midnight sector, and often the fluxes only decrease drastically but do not totally drop out. If magnetospheric satellites enter the tail lobe, which does not contain significant fluxes of energetic particles under normal conditions, the energetic particles would "drop out". Normally, near-earth satellites, such as those with orbits that do not extend beyond geosynchronous altitudes, do not enter the tail lobes. Often the post-dropout recovery of the energetic particle fluxes are associated with the expansion onset of a substorm $/ 5 /$. It is relatively uncommon to observe strong energetic particle flux dropouts in the dawn and dusk sectors $/ 1 /$. Korth et al. $/ 7 /$ has recently reported the occurrence of energetic particle dropouts in the local moming sector and Moldwin et al. $/ 8 /$ have 
observed flux dropouts at nearly all local times and tail lobe entry by geosynchronous satellites from dusk to dawn in the nightside magnetosphere. In this paper we use data similar to that of Korth et al. $77 /$ but extend the study to include all local times reached by CRRES (Combined Release and Radiation Effects Satellite). By using the local magnetic field and particle data in combination we can show the relationship between the particle dropouts and the active current systems at the time of such events.

\section{INSTRUMENTATION}

The CRRES satellite was launched on 25 July 1990 into an elliptical orbit with a geocentric perigee of $\sim 6720 \mathrm{~km}$, apogee of $\sim 39950 \mathrm{~km}$ and an inclination of $18.15^{\circ} .19 /$ The initial local time of apogee was $\sim 8.7$ MLT, and after 1072 orbits (near end of life 14 October 1991) it was at 14 MLT. The orbital inclination in combination with its $\sim 9.6$ hour orbital period and the Earth's tilted magnetic dipole allowed CRRES to reach $L$ shells up to and somewhat beyond $L=8$ on a regular basis. The CRRES near apogee altitude coverage has some gaps in local time. These are the local time intervals near noon on the dayside (from $\sim 09$ through $13 \mathrm{MLT}$ ) and in the early post-midnight region near 01 MLT. The post-midnight coverage gap resulted from the suspension of instrument operations during the CRRES near-apogee eclipse periods.

The instruments used for this study are primarily the Medium Electrons A (MEA) Spectrometer /10/, the Magnetosphere Ion Composition Sensor (MICS) /11/, and the CRRES magnetometer /12/. We have also examined some EPAS (Electron and Proton Wide-Angle Spectrometer) data /13/ and the LEPA (Low Energy Plasma Analyzer) data $/ 14,15$ / to determine whether a plasma signature was present or not. The MEA measures electrons from $\sim 153 \mathrm{keV}$ to $1.5 \mathrm{MeV}$ in 17 differential energy channels /10/, while the MICS measures ions from $\sim 1.2$ to $426 \mathrm{keV} / \mathrm{charge}$ with ion charge and mass composition over mass dependent energy ranges $/ 11 \%$ The MEA and MICS sensors are mounted perpendicular to the CRRES spin axis, which is sun pointed at all times, and cover a relatively wide range of particle pitch angles during a spin period $(\sim 30 \mathrm{sec})$. The EPAS sensor covers the energy ranges of $21-285 \mathrm{keV}$ and $37-3200 \mathrm{keV}$ for electrons and ions, respectively /13/. EPAS has multiple fields of view (FOV) and, by combining the data from the multiple FOV, basically obtains a complete pitch angle distribution every spin period. The LEPA also has multiple FOV and provides complete pitch angle coverage of electrons and ions with energies of 0.12 to $28 \mathrm{keV} / \mathrm{q}$. In the LEPA summary data referenced here $/ 15 /$, only the precipitating and perpendicular plasma electron and ion fluxes were available.

\section{OBSERVATIONS}

For this study we required that the one-minute spin-averaged MEA fluxes, at all energies $\gtrsim 153 \mathrm{keV}$, drop to background levels. This is a rather stringent requirement and basically deletes most of the growth-phase-only signatures /5/ from the study. The MEA criteria were selected because of the easy access to the data and the large geometric factor and good sensitivity of this instrument (see Table 1). The total MEA flux dropout to background levels was chosen to eliminate the more usual flux decreases that occur commonly in the magnetosphere near geosynchronous altitudes. A total of 117 flux dropout events met this requirement between CRRES orbits 76 and 1065 (from 26 August 1990 through 11 October 1991). There were as many as five separate flux dropouts observed in a single orbit. In total, the 117 dropouts were distributed over 70 orbits, as shown in Table 2.

The 117 events were further reduced to those which showed a dropout of the energetic ions $(\geq 20 \mathrm{keV})$. Only 53 events satisfied this criterion. Finally, we selected only those events which showed a dropout of the plasma ions and plasma electrons, respectively, as shown in Table 2. From the initial selection of 117 events, only 20 showed a dropout of all particle fluxes.

Flux dropouts were observed to occur at all local times on the nightside magnetosphere. (We specifically eliminated obvious cases of magnetopause crossings from this study.) The most unexpected regions for them to occur are the local moming and local evening. In these regions the magnetic field topology is usually fairly dipole-like for $\mathrm{L}$ shells below 7 . Thus, we expect that the 
distorted magnetic configurations that lead to flux dropouts should be rarely observed there by

CRRES, even at the moderate latitudes it attains. Also, one would naively expect that the local morning and local evening occurrences should show similar features. We present below one case each of local evening and local moming flux dropouts as examples of the phenomena observed by CRRES before we discuss the statistics of the dropouts. It should be noted that CRRES was in the northem hemisphere near local moming apogee and in the southern hemisphere near local evening apogee.

TABLE 1. Instrument Parameters

\begin{tabular}{c|c|c}
\multicolumn{2}{c|}{ Geometric Factor } & Minimum Measurable Flux \\
\hline MEA & $\begin{array}{c}2.14-5.88 \mathrm{~cm}^{2} \mathrm{sr} \mathrm{keV} \\
\text { [ref. } / 10]\end{array}$ & $-3 \times 10^{-3}$ Electrons $/\left(\mathrm{cm}^{2} \mathrm{sec} \mathrm{sr} \mathrm{keV)}\right.$ \\
MICS & $\begin{array}{c}4.4 \times 10^{-3} \mathrm{~cm}^{2} \mathrm{sr} \mathrm{keV} \\
\text { [ref. } / 11]\end{array}$ & $\sim 23 \mathrm{Ions} /\left(\mathrm{cm}^{2} \mathrm{sec} \mathrm{sr} \mathrm{keV)}\right.$ \\
LEPA & - & $\begin{array}{c}-5 \times 10^{4} \text { particles/(cm } \\
\text { [estimated from summary spectrograms] }\end{array}$ \\
\hline
\end{tabular}

TABLE 2. Particle Flux Dropout Statistics

\begin{tabular}{l|cc|ccc|ccc}
\cline { 2 - 8 } & \multicolumn{2}{c|}{ Number of } & \multicolumn{3}{c|}{ Kp Value } & \multicolumn{3}{c}{ IDst | Value } \\
\cline { 2 - 8 } & Dropouts & Orbits & $<3$ & $3-6$ & $>6$ & $<30$ & $30-100$ & $>100$ \\
\hline Energetic Electrons & 117 & 70 & 17 & 65 & 35 & 34 & 46 & 37 \\
Energetic Ions & 53 & 34 & 6 & 27 & 20 & 13 & 21 & 19 \\
Plasma Ions & 33 & 20 & 0 & 20 & 13 & 5 & 15 & 13 \\
Plasma Electrons & 23 & 14 & 0 & 17 & 6 & 4 & 10 & 9 \\
All Particles & 20 & 14 & 0 & 15 & 5 & 4 & 10 & 6 \\
\hline
\end{tabular}

\section{Local Evening Flux Dropout}

An example of an event which showed the dropout of all particle fluxes on the local evening side of the magnetosphere is presented in Figures 1 and 2. Figure 1 shows the energetic electron data from MEA for this event, which occurred during CRRES orbit 767 on 5 June 1991. The flux from several different electron energies are shown on the plot, with the highest trace being that of the $\sim 153 \mathrm{keV}$ electrons. The decreasing flux levels correspond to increasing energy. The bold trace represents the response of a "background" monitor (BKG) within the MEA. The $\sim 153 \mathrm{keV}$ electron fluxes were required to drop to or below the $B K G$ level before they were identified as a total flux dropout. There were three such periods of total electron flux dropout identified in Figure 1: one near 1500 UT at $\mathrm{L}=7.0,17.8 \mathrm{MLT}$ and $-21.8^{\circ}$ magnetic latitude (MLAT), the second near $1600 \mathrm{UT}$ at $\mathrm{L}=7.4,18.5$ MLT and MLAT $=-21.6^{\circ}$, and the third near 1850 UT at $L=6.0,20.7 \mathrm{MLT}$ and MLAT $=-18.5^{\circ}$.

The lower panels of Figure 2 show energy-time spectrograms of several channels of MICS data for the same orbit. DCR is a measure of the total ion flux independent of species and thus is similar to the measurement made by a total ion plasma instrument such as the LEPA, only at somewhat higher energies ( $7-400 \mathrm{keV} / \mathrm{q}$ versus $0.12-28 \mathrm{keV} / \mathrm{q})$. The MICS " $\geq$ Alpha" channel represents the total flux of all ions with mass $\geq 4$ AMU. The other channels measure the fluxes of the $\mathrm{H}^{+}, \mathrm{He}^{+}, \mathrm{He}^{++}$ and $\mathrm{O}^{+}$above a mass dependent energy threshold $/ 11 \%$. There are periods in the MICS data where essentially all ions dropped out, especially during the $1600 \mathrm{UT}$ event. Note that each $\mathrm{O}^{+}$spectrum has been averaged over 8 minutes, which means there were a few counts in each interval that straddles 


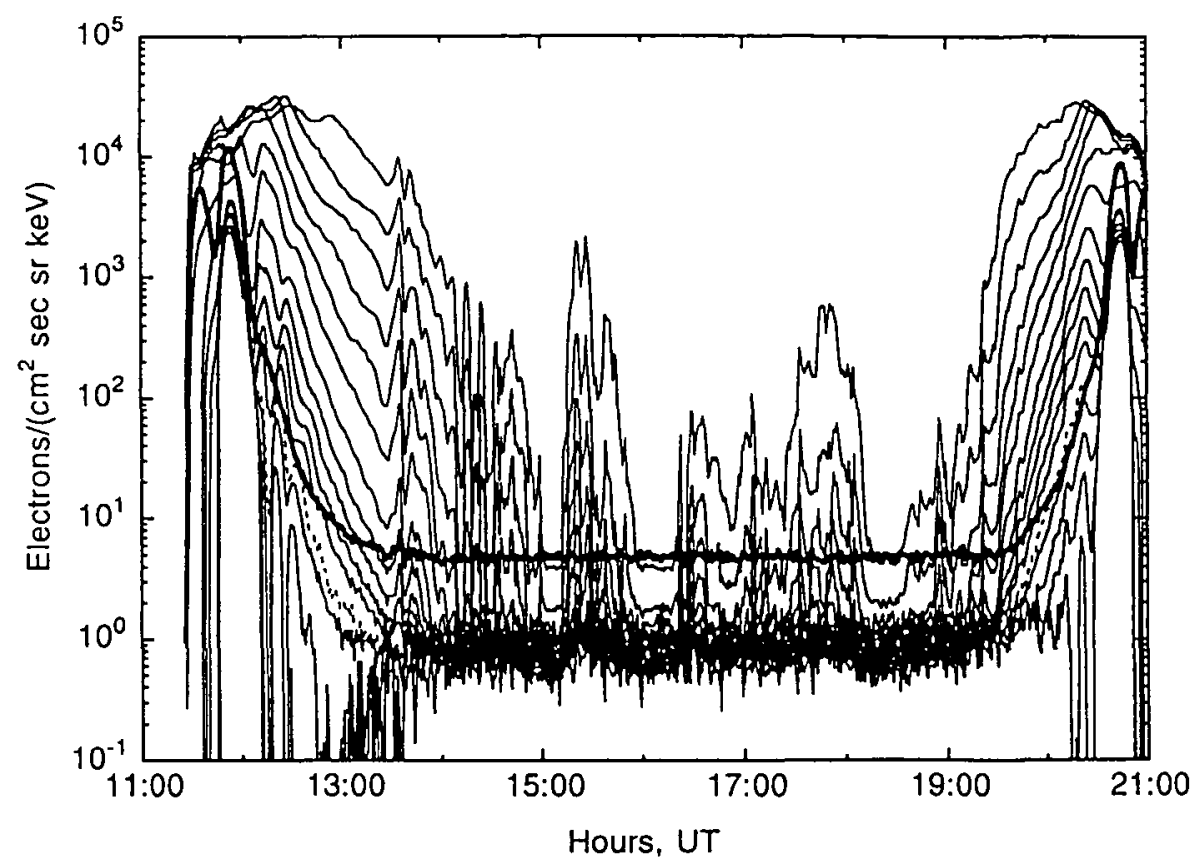

Fig. 1. Energetic electron fluxes from the CRRES MEA sensor during orbit 767 on 5 June 1991. The topmost trace is the flux of $\sim 153 \mathrm{keV}$ electrons and the succeeding traces are for successively higher energies /9\%. The bold line represents the response to a background monitor (BKG). The $\sim 153 \mathrm{keV}$ electron fluxes were required to be at or below BKG in order to qualify as a flux dropout for this study. For this period, the energetic electron flux dropouts occurred at 54100,57600 and $67800 \mathrm{sec}$ (or 1500, 1600 and $1850 \mathrm{UT}$ ).

the flux dropout's edges. It should be noted that the $\mathrm{He}^{+}$flux dropped well before the rest of the ions and that it recovered later than the other ions too. This relationship between the two charge states of Helium was often observed in the flux dropout events. This would be expected if $\mathrm{He}^{++}$is presumed to be the dominant $\mathrm{He}$ ion in the outer magnetosphere, as is expected for a solar wind source in which $\mathrm{He}^{+}$is generated via charge exchange from $\mathrm{He}^{++}$. The $\mathrm{He}^{+}$would be most prevalent on the lower $\mathrm{L}$ shells deep inside the plasma sheet. If the flux dropout were the result of a reconfiguration of the magnetosphere that caused the CRRES satellite to be on field lines that thread the distant tail near the interface between the plasma sheet and tail lobe field lines, then the ion composition just prior to and just after the flux dropout should reflect that of the distant plasma sheet, as was apparently the case for this event.

The upper three panels of Figure 2 show the magnetometer measurements for this event. The data are presented as the differences between the Tsyganenko 1987 field model $/ 16 /$ for $\mathrm{KP}=3$ and the observed values. The direction $(\hat{b}$ ) was taken as the Tsyganenko field direction. The reference coordinate directions $\hat{b}, \hat{\mathbf{r}}$ and $\hat{w}$ are in the directions parallel to the Tsyganenko field, earthward in the plane containing $\hat{b}$ and the radius vector from the center of the earth to CRRES and westwards, respectively, as shown in Figure 3.

$\partial \mathrm{Bb}, \partial \mathrm{Br}$ and $\partial \mathrm{Bw}$ are the differences (residuals) between the measured and the Tsyganenko model field. As can be seen by the magnitudes of these components, especially $\partial \mathrm{Br}$, the magnetic field intensity at CRRES was stronger than normal and very "tail-like". There was also a significant, but varying, westward component. The large $\partial \mathrm{B}_{\mathrm{r}}$ and $\partial \mathrm{B}_{\mathrm{W}}$ indicate that strong currents were flowing in the neighborhood of CRRES. Specifically, near the onsets of the particle dropouts, the field became more tail-like and rotated from a westward to an eastward direction. At each flux recovery the field became less tail-like (although still much more tail-like than normal) and rotated back towards the meridian plane (where $\partial \mathrm{Bw}=0$ ). In particular, a strong dipolarization occurred in conjunction with the ion flux recovery near 1515 UT. A second (momentary) and third dipolarization occurred near $1620 \mathrm{UT}$, where there was first a short ion flux recovery with subsequent dropout followed by a permanent ion flux recovery near 1628 UT (see DCR in Figure 2 near 15200-1530 UT). These field 


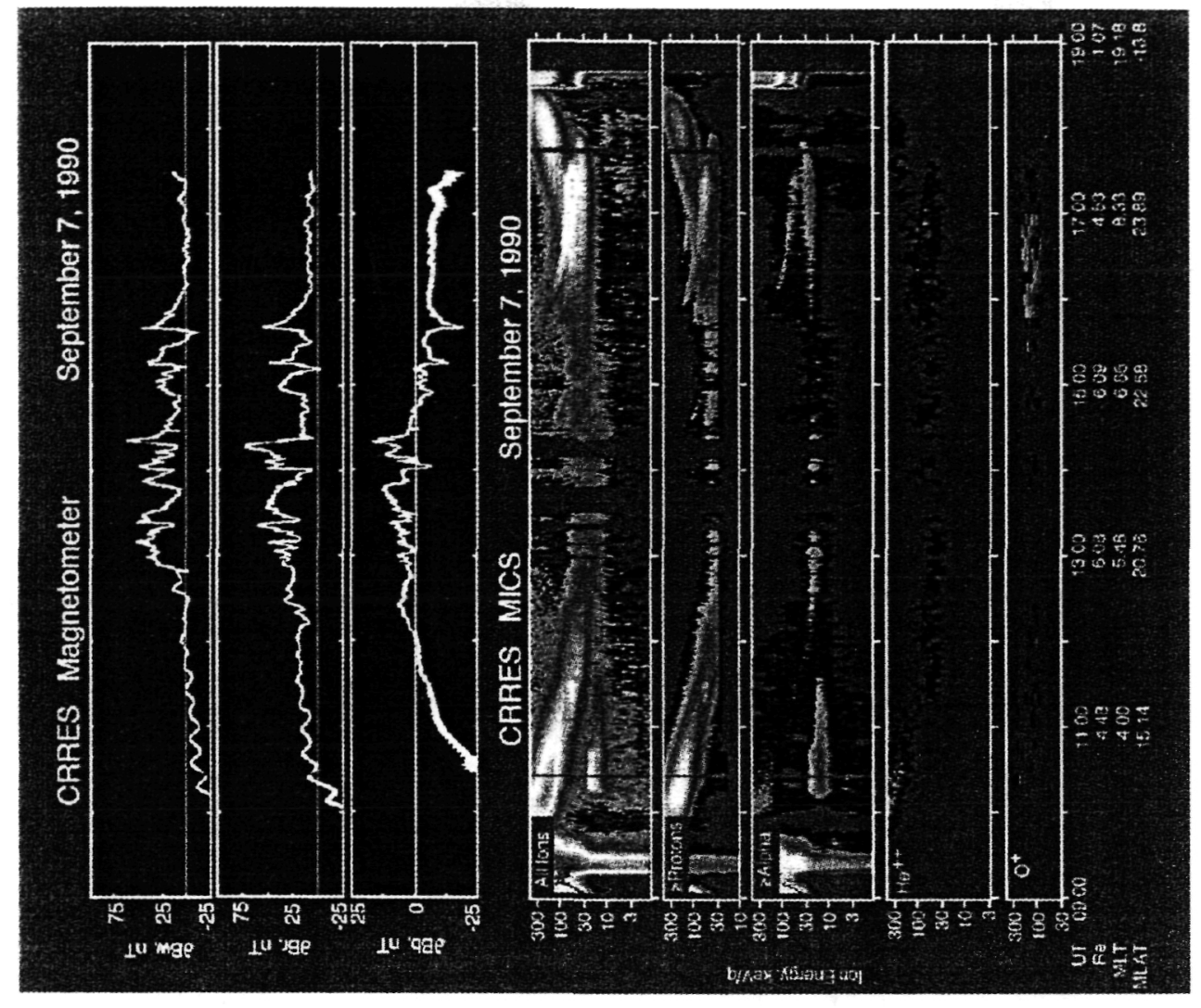

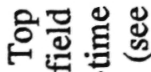

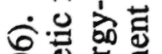

으형 톨

현 焉

녕 흥.

픙 年

ूั

氠肕

월

면를

के ठ․ㅇ ్ㅐ

$\sim$ ऽ 불

뎡녈웛

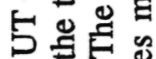

음

ร่ ๕

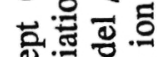

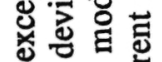

N

일

일 卷

江 露 芲先

戸 蛋

เ 2 은

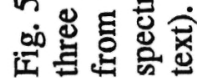

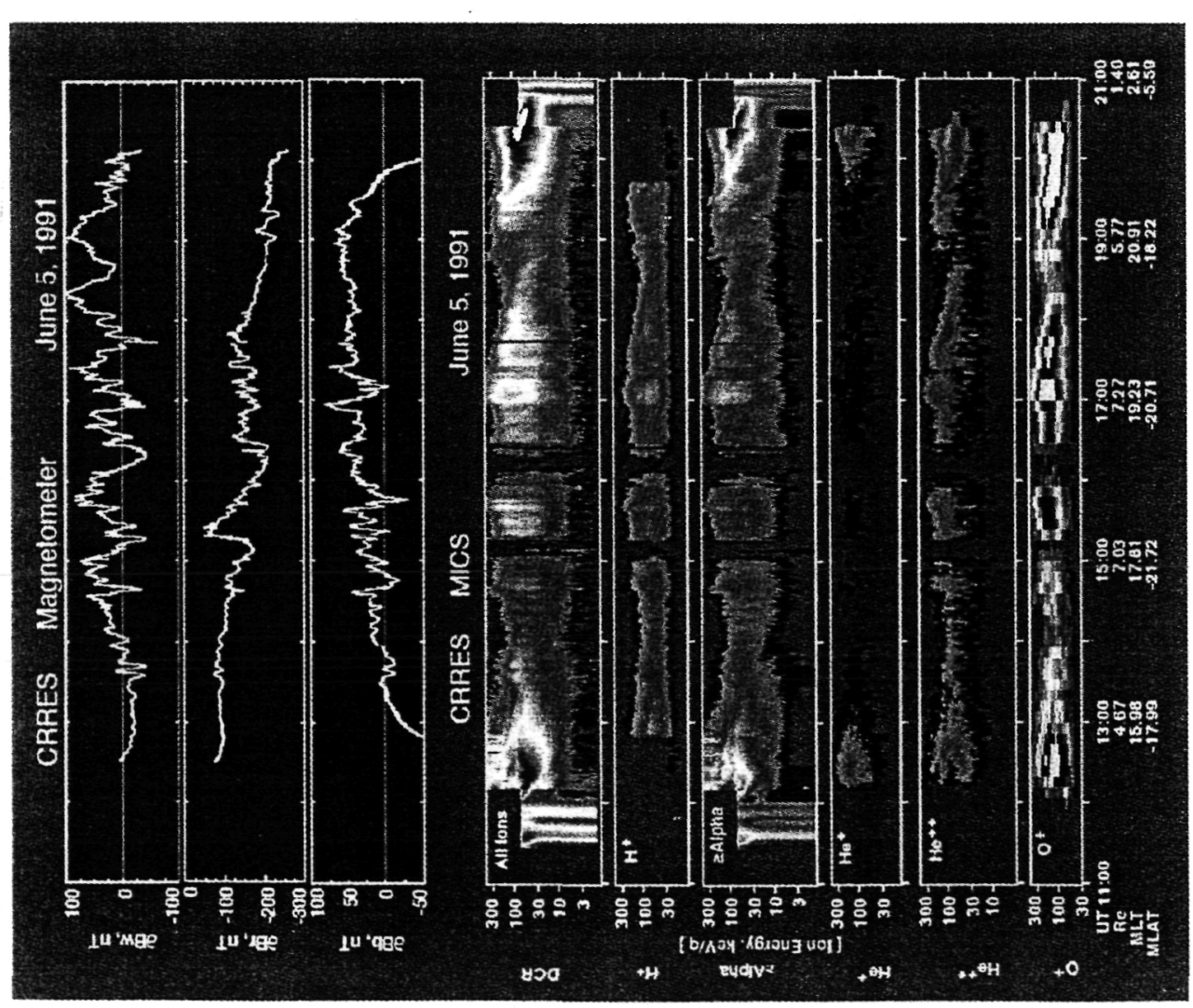

증 岁焉

ํㅠㄹ

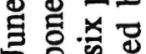

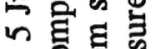

ㄷㅇㅇㅇㅀ

웡요

氙心

त) है

至㟧宑

훙 을등

栗票

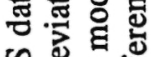

บ 응

之严䞤

至势

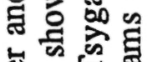

包的舫

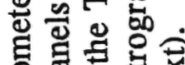

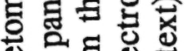

\&

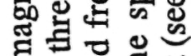

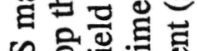

论句密

․ㅠ

论它㐘

도

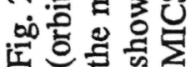


signatures are consistent with the CRRES satellite having been approached and crossed by a Region 1

current system as the particle fluxes dropped out and then recovered.

While there was a total energetic electron flux dropout near $1850 \mathrm{UT}$, there was not a comparable dropout in the energetic ion fluxes. The intensification of the ion fluxes near $1700 \mathrm{UT}(61200 \mathrm{sec})$ occurred simultaneously with a weak energetic electron recovery. The ion fluxes returned to lower levels by 1730 UT, which is just prior to the electron flux recovery that peaks near 1752 UT.

The plasma data (not displayed here) showed a total plasma electron dropout in conjunction with both of the energetic ion dropouts in Figure 2. The plasma ions also dropped out but only just prior to the energetic particle flux recoveries near 1515 UT and 1625-1628 UT. Both the plasma electrons and ions showed the same momentary flux recovery, subsequent short dropout and final recovery during this latter period, as was observed in the energetic ions (ref. Figure 2). Like the energetic ions, the plasma data did not show a flux dropout near 1850 UT and indicated that CRRES remained in a hot plasma sheet plasma. The only other significant feature in the plasma data was the occurrence of low energy $(<1 \mathrm{keV})$ field-aligned electron fluxes just prior to and immediately after the plasma and energetic particle dropouts discussed above.

\section{Local Morning Flux Dropout}

Figure 4 shows an example of energetic electron total flux dropouts observed by CRRES near local morning on 7 September 1990. There were five flux dropout intervals (marked by shaded vertical bars in Figure 4) which started near 1300, 1308, 1320, 1332 and 1409 UT. We have plotted the CRRES magnetic difference data (as discussed above) on the same plot for comparison. Note that the $\partial \mathrm{Br}$ was positive when the magnetic field had tail-like conditions because CRRES was in the northem hemisphere, whereas $\partial \mathrm{Br}$ was negative during the local evening tail-like conditions discussed above.
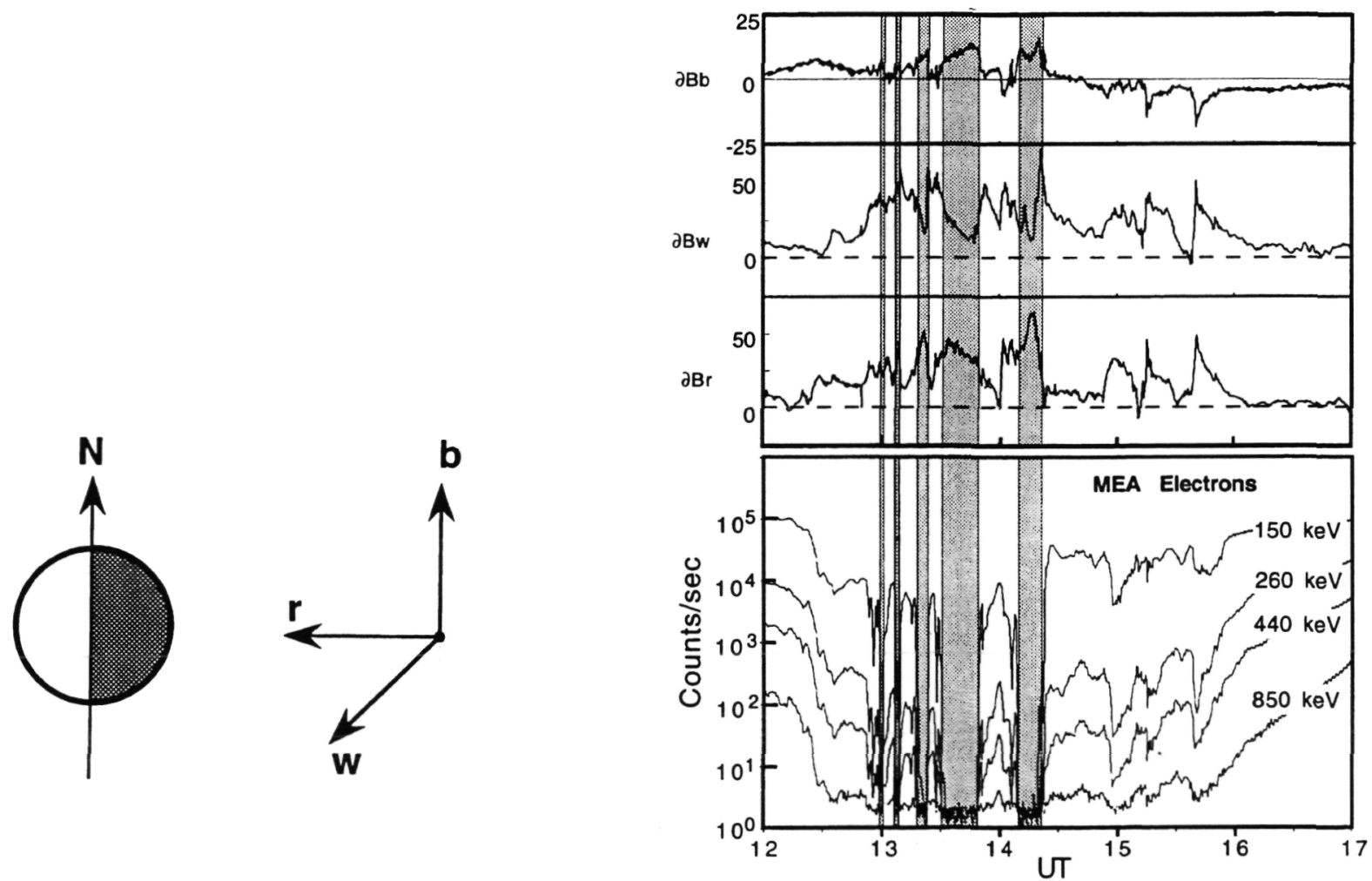

Fig. 3. Coordinate system used for detrended magnetic field data. $\hat{b}$ is parallel to the Tsyganenko field vector, $\hat{\mathbf{r}}$ is earthward in the plane defined by $\hat{b}$ and the radius vector from the center of the earth to CRRES and $\hat{w}$ is westward.

Fig. 4. Energetic electrons and detrended magnetic field data for 7 September 1990 (CRRES orbit 106). The flux dropout onsets are 1300, 1308, 1320, 1332 and 1409 UT. 
In.both cases, the $\partial \mathrm{Bw}$ tumed from westward towards zero or eastward at the flux dropout onsets and retumed westward at the flux recovery. Like the local evening observations, the field is compressed (positive $\partial \mathrm{Bb}$ ) during the dropouts. Also, the field was tail-like at the dropout onset and dipolarized at the flux recovery. These signatures, like those of the local evening dropouts, are consistent with CRRES having been approached and crossed by a region 1 current system at the flux dropout and at recovery. This would be consistent with CRRES having entered the tail lobe on the local morning side of the magnetosphere.

Figure 5 shows the MICS energetic ion data and, again, the magnetic field difference plots for this event. In this case, there was a one-to-one correspondence between the energetic electron flux dropouts and the energetic ion flux dropouts. The primary difference between Figure 5 and Figure 2 was the relative absence of $\mathrm{O}+$ ions throughout the magnetosphere on 7 September. Other than this lack of $\mathrm{O}^{+}$and $\mathrm{He}^{++}$ions on 7 September, flux dropout features were remarkably identical for these two events that were observed on opposite sides of the magnetosphere.

Examination of the plasma data (not shown here) showed that there was a one-to-one correspondence between the energetic particle flux dropouts and the plasma dropouts for the 7 September events. The narrow dropouts at 1300 and 1308 UT were barely resolved in the summary plasma electron plots and somewhat less clear in the ion plots. Like the local evening case, intense low energy fieldaligned electron fluxes were observed just prior to and immediately after the flux dropouts. In general, such field-aligned "soft" electron fluxes were observed near the majority of the energetic ion flux dropouts and at nearly all of the plasma dropouts.

\section{Flux Dropout Statistics}

As mentioned above, we examined the energetic ion, magnetic field and plasma data that were available for all 117 energetic flux dropout events. Table 2 summarizes the breakdown into the different categories according to whether the energetic ions and plasma showed flux dropouts and some crude ranges of $K_{P}$ and $D_{S T}$ levels for the corresponding events. In general, the $K_{P}$ values observed at the time of the flux dropouts were moderate to high. This is presented graphically in Figure 6, where the distributions of both energetic electron and energetic ion flux dropouts in $K_{P}$ and $D_{S T}$ are shown.The dominance of moderate to high $\mathrm{KP}$ values indicates the flux dropouts occur during disturbed periods, as would be expected for the strong currents observed in association with the dropouts (e.g., see Figures 2 and 5). But, there is no strong dependence on DST The majority of the flux dropouts occurred during relatively low or modest DST This is somewhat surprising. It indicates that magnetic storm conditions are not necessary for the flux dropouts to occur. One might have expected that flux dropouts would have been more prevalent during the magnetically disturbed conditions generally associated with larger ring current enhancements.

Figures 7 and 8 show the distribution of the CRRES flux dropouts in magnetic local time and $L$ and in magnetic latitude and $\mathrm{L}$, respectively. Note that the paucity of events in two different local time regions, one post-midnight ( 0 - 2 MLT) and one near noon (9-14 MLT), partially reflects the lack of coverage in these regions. [CRRES apogee never reached the near noon sector and the instruments were turned off in the post-midnight sector during the eclipse season.] There was only one obvious magnetopause crossing observed by CRRES and this was eliminated from the data set. The different panels in Figures 7 and 8 correspond to the distribution of energetic electron dropouts (a), the energetic ion dropouts (b) and the plasma ion dropouts (c), respectively. Note that when one required that the plasma ions disappear to have a total particle dropout, there remained only two events that met this criterion on the moming side but a significant number on the evening side. All flux dropout events were observed at magnetic latitudes $10^{\circ}$ or more above and below the magnetic equator. The preponderance of the dropouts occurred for $L>6.6$ and all but two occurred for $L>5.5$. This indicates that to have a good probability of observing particle dropouts requires a satellite to be at or above geosynchronous altitude and/or at moderate to high magnetic latitudes.

The trend of the points in Figure 8 to move to higher latitude with larger $\mathrm{L}$ shell is a result of the CRRES orbital configuration. As noted in the introduction, CRRES only reached $\mathrm{L} \leq 6.3$ at the 

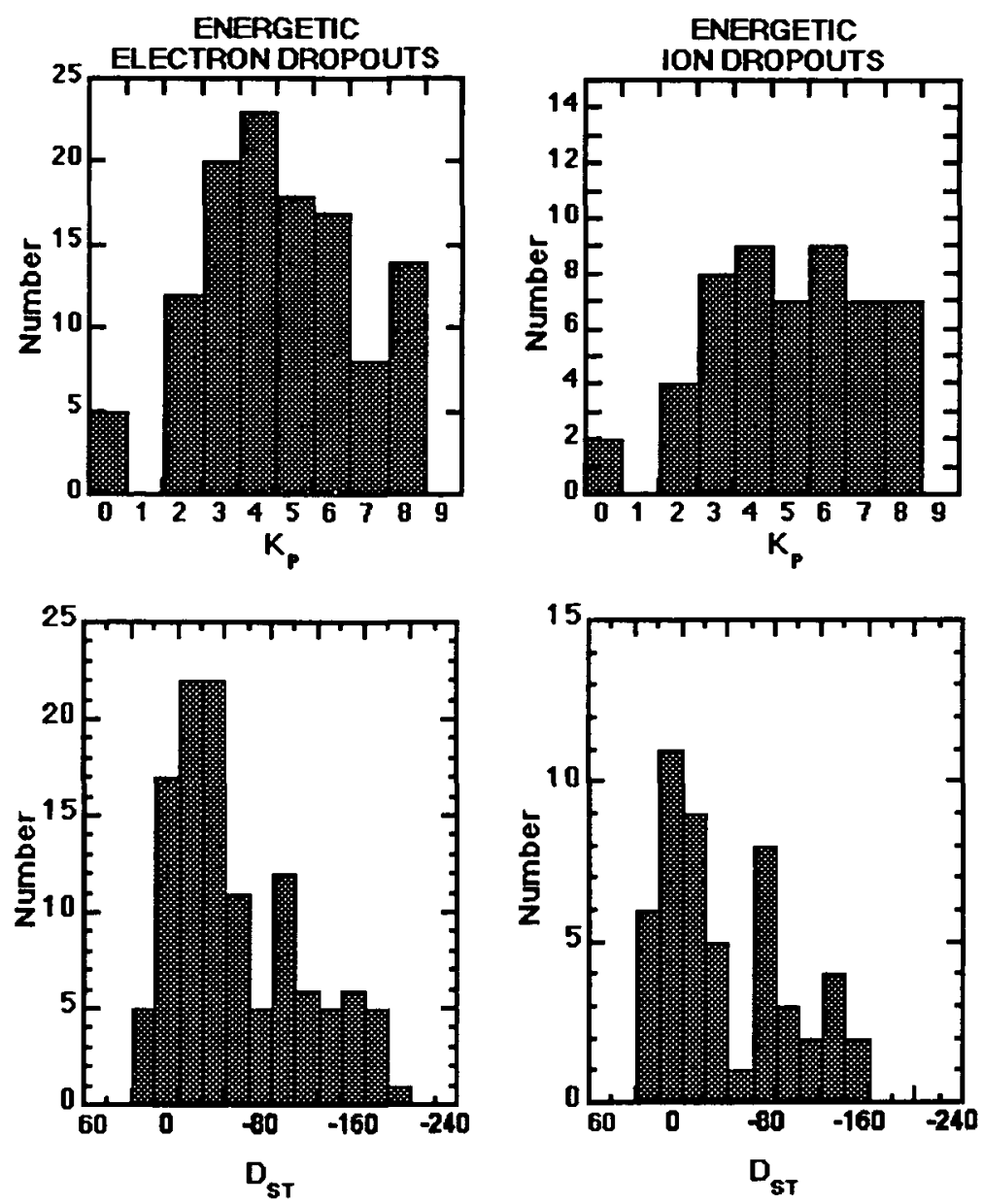

Fig. 6. Energetic ion and electron dropout occurrence frequency versus $K_{P}$ and DST.

magnetic equator, but because of its moderate $18^{\circ}$ inclination it could reach higher $\mathrm{L}$ shells when apogee was off the magnetic equator. The $\sim 10$ hour orbital period allowed the CRRES apogee to pass through a significant range of magnetic latitudes on a weekly basis. The moming side data were taken in the late summer and fall of 1990 while the midnight to dusk data were taken in the spring through summer of 1991. The combination of Figures 7 and 8 show that the morning side events all occurred in the northem hemisphere and the midnight through dusk events occurred in the southern hemisphere.

Finally, we looked at the changes in the local magnetic field at the times of the total plasma dropout onsets to see if they were all consistent with the picture, described above, of the CRRES satellite crossing (or being crossed by) a Region 1 current system, entering the tail lobes and then retuming at flux recovery. We grouped the events into morning side and dusk side local time regimes, as shown in Table 3. We examined in detail the pre- and post-dropout field trends and the changes that occurred at flux dropout onset and recovery. We categorized the changes, as shown in Table 3 , according to whether the field was tail-like (had a significant radial component) or was becoming taillike at onset and whether the azimuthal component changed and in which direction. In all cases, the field was either strongly tail-like or became tail-like at flux dropout onset. In the majority of cases, the azimuthal component tumed eastward at onset. Since all the moming side events occurred in the northem hemisphere and the dusk side events were in the southem hemisphere, an eastward rotation of the field would be consistent with crossing a Region 1 current system in the respective local time sectors. So it is clear from Table 3 that the majority of total plasma dropout events occurred as the CRRES satellite crossed (or was crossed by) the boundary between the plasma sheet and tail lobe as evidenced by the passage of the Region 1 current system along with the strongly tail-like field geometry and the loss of all particle fluxes. 


\begin{tabular}{|c|c|c|c|c|c|c|c|c|c|}
\hline \multirow[t]{5}{*}{ ' } & \multirow[t]{5}{*}{ 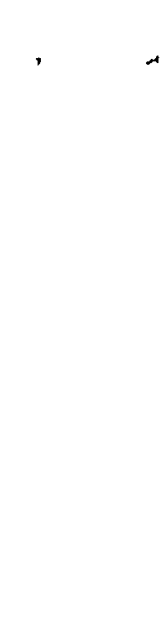 } & \multirow[t]{2}{*}{ • } & TABLE 3. & \multicolumn{6}{|c|}{ Field Changes During Total Plasma Dropout Onsets } \\
\hline & & & & $\partial \mathbf{B} \mathbf{w}$ & & & $\partial \mathbf{B r}$ & & \\
\hline & & MLT & Eastward & Westward & $\begin{array}{c}\text { No } \\
\text { Change }\end{array}$ & $\begin{array}{c}\text { More } \\
\text { Tail } \\
\text { Like }\end{array}$ & $\begin{array}{l}\text { Less } \\
\text { Tail } \\
\text { Like }\end{array}$ & $\begin{array}{l}\text { No** } \\
\text { Change }\end{array}$ & $\begin{array}{c}\text { Numbe } \\
\text { of } \\
\text { Events }\end{array}$ \\
\hline & & $3-9$ & 5 & 1 & -. & 4 & - & 2 & 6 \\
\hline & & $15 \cdot 21$ & 11 & 1 & 1 & 10 & -. & 3 & 13 \\
\hline
\end{tabular}

** $\partial \mathrm{Br}$ remained large but constant

(a.) Local Time Distribution of Energetic Electron Total Flux Dropouts

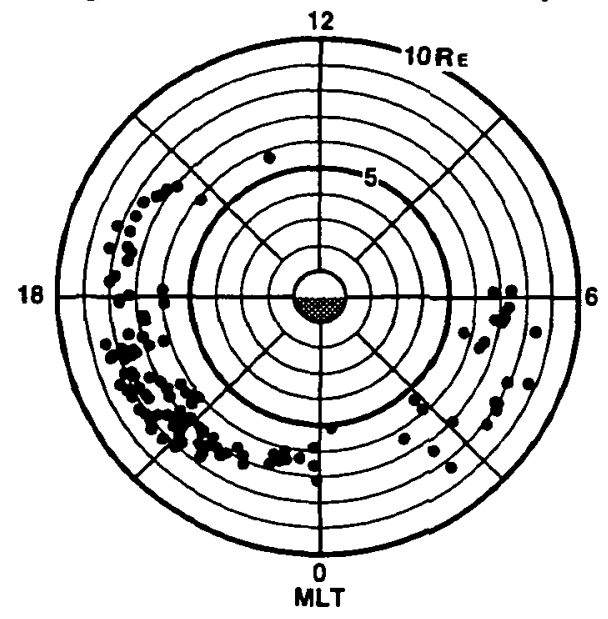

(c.) Local Time Distribution of Energetic and Plasma Ion Total Flux Dropouts

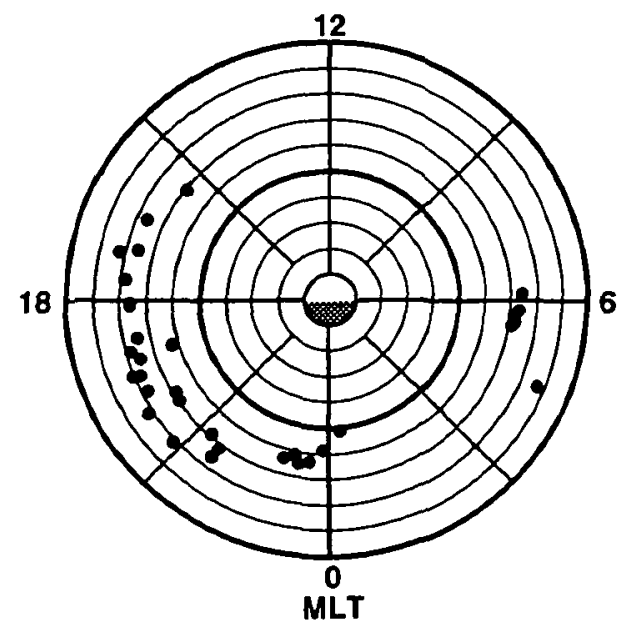

(b.) Local Time Distribution of Energetic lon Total Flux Dropouts

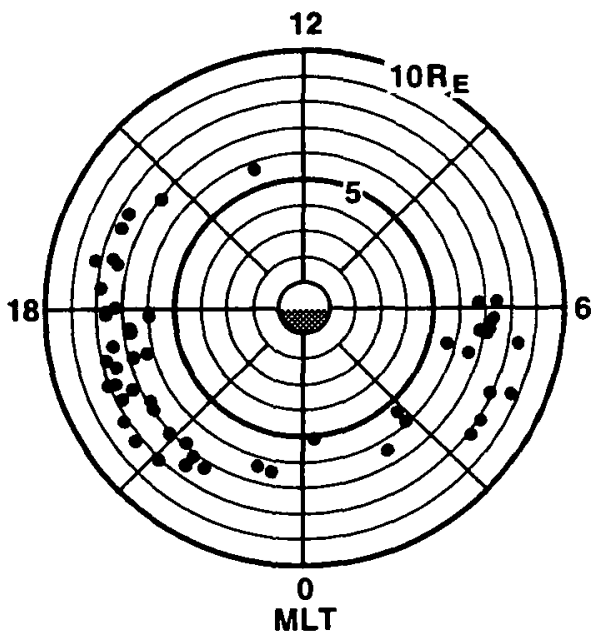

Fig. 7. Distribution of flux dropouts in magnetic local time and L shell: (a) for engergetic electrons, (b) for energetic ions, and (c) for plasma ions. 

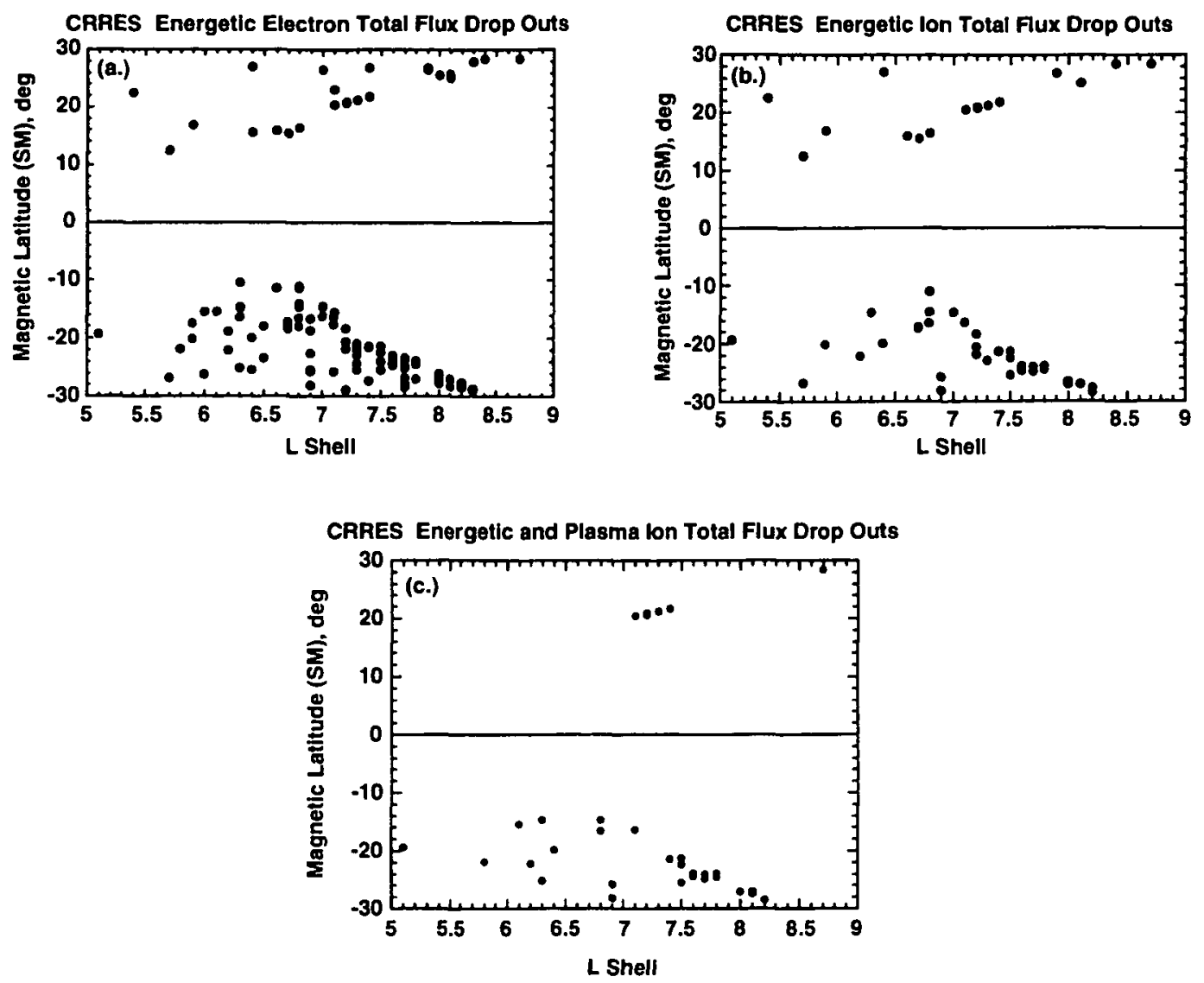

Fig. 8. Distribution of flux dropouts in magnetic latitude and $\mathrm{L}$ shell: (a) for energetic electrons, (b) for energetic ions, and (c) for plasma ions.

\section{SUMMARY AND CONCLUSIONS}

The local morning and local evening flux dropout events described here and the total plasma dropouts represented in Table 3 clearly show that CRRES entered the magnetotail lobe during these flux dropouts. In general, as seen in the two events shown, the recovery from the flux dropouts was not associated with a signature of substorm expansion onset at CRRES. Given that most of the events were observed away from the midnight sector, it is possible that the substorm signature was not observable by CRRES. To determine whether there was a substorm associated with these events requires examining ground magnetometer and other satellite data, which is beyond the scope of the present work, but will be done as part of a continuing effort on this subject (see Korth et al. $7 /$ ).

We emphasize that care was taken to eliminate magnetopause crossings (only one found) from this data set by examining the plasma data for signatures of magnetosheath plasma. In all cases, very significant currents were flowing near the CRRES satellite as evidenced by the strong changes in the magnetic field topology from average conditions. The field was generally tail-like, indicating that the satellite was near the inner edge of the cross-tail current system. The actual current system configuration that can give rise to such strongly tail-like conditions as were observed near 5.5-6.5 MLT (see Figure 5) and 17.8-19.0 MLT (see Figure 2) is not clear, and its details require more observational constraints than are provided by a single satellite measurement.

It should be noted that the majority of the energetic particle flux dropouts included in this data set had rapid onsets like those shown in Figures 1, 2, 4 and 5. The criterion that the lowest energy channel from the CRRES MEA sensor must drop to background levels removed most of the events that had slowly decreasing growth-phase-like flux decrease signatures $/ 5 /$. While this reduced the total 
number of electron flux dropout candidates, it provided a list of those most likely to also show energetic ion and plasma dropouts. The high sensitivity of both the energetic electron and ion sensors (MEA and MICS, see Table 1) allowed us to set very stringent minimum flux levels for our dropout identifications. As can been seen in Figures 1,2,4 and 5, these thresholds were orders of magnitude below typical magnetospheric levels and comparable to the cosmic ray background response of the sensors. It should also be noted that all particle data in this study were averaged over one or more satellite rtations so that details of the particle angular distributions were not visible.

The difference between the number of energetic electron and energetic ion flux dropouts is most likely a result of the ions greater gyro-radii. That is, the ions are observable within an ion gyroradius of the plasma-sheet/tail-lobe boundary on the tail lobe side of the boundary, which is much deeper in the tail lobe than an energetic electron can reach. In a follow-on study we will examine the energetic ions and electrons in greater detail and use the available angular distribution data /13/ to "sound" the boundary in the neighborhood of the flux dropout intervals. This should help to more clearly delineate the differences between the energetic electrons and ions during the flux dropout events. It is most likely that, in some cases, the satellite approached to within a particle gyro-radius of the boundary but did not pass through it.

The local time and seasonal results described above are in apparent disagreement with the recent results of Moldwin et al. for events observed at geosynchronous orbit, wherein they found a preference for the southem hemisphere dropouts to occur during the winter season and the northern hemisphere dropouts to occur during the summer season /8/. In our case, the season did not appear to matter. But, the Moldwin et al. results were from data taken near the geographic equator, whereas the CRRES orbit sampled a much wider range of geographic and geomagnetic latitudes. Therefore, CRRES observed the dropouts only when it was more than $10^{\circ}$ away from the geomagnetic equator. We did not note the geographic position of the dropouts during this study. This will be done during the expanded study noted above.

At this point we do not know whether the field-aligned plasma electrons, observed at the edges of the flux dropouts, are moving towards or away from the earth since the actual direction is not indicated in the summary data. This will be examined in greater detail in a later paper. It is important because it will more clearly identify the plasma regime that CRRES was immersed in just prior to the flux dropouts and at the recovery. Given the strong field-aligned currents detected by the magnetometer in these regions, it is possible these intense electrons may be the current carrying particles.

It is clear from the above discussion that much remains to be done on the subject of flux dropouts. Some of the questions that arise are: How can the field be so tail-like at dusk and dawn on such low L shells $(\leq 7)$ ? Why are the electron dropouts more prevalent than ion dropouts? What are the motions of the tail-lobe boundary relative to CRRES at the times of the dropouts? What is the configuration of the tail-lobe boundary and the corresponding current systems during such events? Were the solar wind conditions extreme (i.e., was the magnetosphere compressed)? Were substorm onsets associated with many of the events? Were such flux dropout events observable at other spacecraft, such as those in geosynchronous orbit. The answer to these and other questions will be the focus of our future work on this subject.

\section{ACKNOWLEDGMENTS}

We would like to thank D. Hardy, K. Kerns and the U. S. Air Force Phillips Laboratory/GPSP for producing the LEPA data and N. Watkins and A. Johnstone for making it available for this study. We would also like to thank G. Reeves, $M$. Thomsen and $M$. Moldwin for useful discussions on the topic of flux dropouts. The EPAS and MICS instruments were designed and constructed with support from the Max-Planck-Gesellschaft zur Förderung der Wisenschaften. The MEA instrument and the MEA, MICS and EPAS digital data processors were designed and constructed at The Aerospace Corporation. The work performed at The Aerospace Corporation was supported in part by the U.S. Air Force System Command's Space System Division under contract No. F04701-91-C-0089. 


\section{REFERENCES}

1. Walker, R. J. , K. N. Erickson, R. L. Swanson, and J. R. Winckler, Substorm-associated particle boundary motion at synchronous orbit, J. Geophys. Res., 11, 5541, (1976)

2. Russell, C. T., On the occurrence of magnetopause crossings at $6.6 \mathrm{Re}$, Geophys. Res. Lett., 3, 593 (1976)

3. Studemann, W. B. Wilken, D. N. Baker, P. R. Higbie, R. D. Belian, and T. A. Fritz, Planet. Space Sci., $\underline{34}, 825$ (1986)

4. Baker, D. N. Multipoint measurements of energetic particles in the magnetosphere, Adv. Space Res., 8, \#9, 385 (1988)

5. Baker, D. N., and R. L. McPherron, Extreme energetic particle decreases near geostationary orbit: A manifestation of current diversion within the inner plasma sheet, J. Geophys. Res., 95 , 6951-6599 (1990)

6. Baker, D. N., P. R. Higbie, E. W. Hones and R. D. Belian, High resolution energetic particle measurements at $6.6 \mathrm{Re}$, Low-energy electron anisotropies and short-term substorm predictions, $J$. Geophys. Res., 83, 4863 (1978)

7. Korth, A., R. Friedel, D. N. Baker, H. Luhr, S. L. Ullaland, J. F. Fennell, and G. D. Reeves, Dynamics of the plasma sheet in the dawn sector of the magnetosphere: Observations from CRRES, to be published, Proceedings of ICS-2 (1994).

8. Moldwin, M. B., M. F. Thomsen, S. J. Bame, D. J. McComas, J. Birn, G. D. Reeves, R. Nemzek and R. D. Belian, Flux Dropouts of Plasma and Energetic Particles at Geosynchronous Orbit During Large Geomagnetic Storms: Entry into the Lobes, submitted to J. Geophys. Res., (1994)

9. M. H. Johnson and J. Kierein, Combined Release and Radiation Effects Satellite (CRRES): Spacecraft and Mission, J. Spacecraft and Rockets, 29. 556, (1992).

10. Vampola, A. L., J. V. Osborn, B. M. Johnson, CRRES Magnetic Electron Spectrometer AFGL-701-5A (MEA), J. Spacecraft and Rockets, 29, 592 (1992).

11. Wilken, B., W. Weiss, D. Hall, M. Grande, F. Soraas, J. F. Fennell, Magnetospheric Ion Composition Spectrometer Onboard the CRRES Spacecraft, J. Spacecraft and Rockets, 29, 585 (1992).

12. H. J. Singer, W. P. Sullivan, P. Anderson, F. Mozer, P. Harvey, J. Wygant, W. McNeil, Fluxgate Magnetometer Instrument on the CRRES, J. Spacecraft and Rockets, 29, 599 (1992).

13. Korth, A., G. Kremser, B. Wilken, W. Guttler, S. L. Ullaland, R. Koga, Electron and Proton Wide-Angle Spectrometer (EPAS) on the CRRES Spacecraft, Journal of Spacecraft and Rockets, 29, 609 (1992).

14. Hardy, D, Low Energy Plasma Analyzer (LEPA) on CRRES, Nucl. Inst. and Methods, 1993.

15. Kerns, K. J., D. A. Hardy, and M. S. Gussenhoven, Modeling of Convection Boundaries Seen by CRRES in 120-eV to $28 \mathrm{keV}$ Particles, J. Geophys. Res., 99. 2403, (1994).

16. Tsyganenko, N. A., Global Quantitative Models of the Geomagnetic Field in the Cislunar Magnetosphere for Different Disturbance Levels, Planet. Space Sci. 35, 1347, (1987). 